\title{
Е.Б. Грибанова
}

\section{Методы решения обратных задач экономического анализа с помощью минимизации приращений аргументов}

\begin{abstract}
Предложен метод решения обратных задач экономического анализа при минимальном изменении аргументов. Его реализация является более простой по сравнению с методами решения нелинейных оптимизационных задач. Рассмотрена аддитивная, мультипликативная и кратная модель. В качестве примера приведена задача формирования рейтинга группы онлайновой социальной сети.
\end{abstract}

Ключевые слова: обратные вычисления, оптимизация, квадратичное программирование, рейтинг. doi: $10.21293 / 1818-0442-2018-21-2-95-99$

При принятии решений в области экономики специалист сталкивается с прямыми и обратными задачами. Под решением задач с помощью обратных вычислений [1-3] понимают нахождение приращений аргументов функции на основе следующей информации:

- начальные значения аргументов $\left(x_{1}, x_{2}\right)$ и функции ( $y$ );

- новое значение функции ( $y \pm \Delta y)$;

- коэффициенты относительной важности аргументов $(\alpha, \beta)$;

- направление изменений аргументов $(+,-)$.

Таким образом, может быть сформирована система уравнений:

$$
\left\{\begin{array}{l}
y \pm \Delta y=f\left(x_{1} \pm \Delta x_{1}(\alpha), x_{2} \pm \Delta x_{2}(\beta)\right) ; \\
\frac{\Delta x_{1}}{\Delta x_{2}}=\frac{\alpha}{\beta} \\
\alpha+\beta=1 .
\end{array}\right.
$$

Решением системы будут величины $\Delta x_{1}$ и $\Delta x_{2}$, обеспечивающие значение результирующего показателя, равное $y \pm \Delta y$.

Полученные значения могут быть использованы для определения направлений изменения показателей деятельности исследуемого объекта для достижения заданной цели. Примеры решения задач такого рода рассмотрены в статьях [4-7]. В работе [8] метод обратных вычислений был использован совместно с лагранжевым анализом конечных изменений, что позволило выявить факторы, оказавшие наибольшее влияние на результат. При наличии ограничений на величины аргументов могут быть использованы итерационные алгоритмы, в том числе с применением элементов случайного поиска $[3,9]$.

Результат решения системы (1) определяется значениями коэффициентов относительной важности и направлением изменений показателей, которые устанавливаются исследователем. Привязка к мнению эксперта имеет свои положительные стороны: может быть рассмотрено несколько возможных вариантов решения задачи, коэффициенты могут быть установлены с учётом реальной возможности направления изменения аргументов и их взаимозави- симости. Полученное решение впоследствии может быть скорректировано с учетом дополнительных условий. Так, например, в работе [10] рассматривается получение решения в соответствии с «золотыми» пропорциями показателей. Однако иногда возникает необходимость получить результат без привлечения экспертной информации. К такому случаю можно отнести нахождение решения, максимально близкого к исходному, т.е. при минимальном изменении значений аргументов.

В данной работе рассмотрено решение задач с помощью обратных вычислений в случае минимального приращения аргументов (без привлечения экспертной информации). Модификация классической схемы решения (1) при этом выражается в изменении величины соотношения значений приращений аргументов, которая будет определяться теперь угловым коэффициентом линии уровня, определяемого новым значением функции. Далее будет рассмотрено решение задачи в случае аддитивной, кратной и мультипликативной зависимости.

\section{Аддитивная модель}

Рассмотрим задачу с аддитивной зависимостью (например, суммарные затраты равны сумме постоянных и переменных затрат [1]):

$$
y=x_{1}+x_{2} \text {. }
$$

Пусть начальные условия задачи: $y^{0}=5, x_{1}^{0}=2$, $x_{2}^{0}=3$. Необходимо определить такие значения $x_{1}^{1}$, $x_{2}^{1}$, при которых $y^{1}$ равно 10 . Построим две линии уровня 5 и 10 соответственно (рис. 1), представляющие собой параллельные прямые. Точка А соответствует начальному условию задачи. Точки линии $x_{2}=10-x_{1}$ обеспечат значение результирующей величины, равное 10 . Точки $B$ и $C$ соответствуют случаям, когда искомое значение функции будет получено только за счет изменения $x_{2}$ и $x_{1}$ соответственно. Точки линии $x_{2}=10-x_{1}$, принадлежащие отрезку $B C$, будут получены при увеличении аргументов функции $\left(x_{1}, x_{2}\right)$, расположенные левее точки $B$ - при уменьшении аргумента $x_{1}$ и увеличении $x_{2}$ $\left(x_{1}^{-}, x_{2}^{+}\right)$, а точки правее $C-$ при увеличении $x_{1}$ и уменьшении $x_{2}\left(x_{1}^{+}, x_{2}^{-}\right)$. 


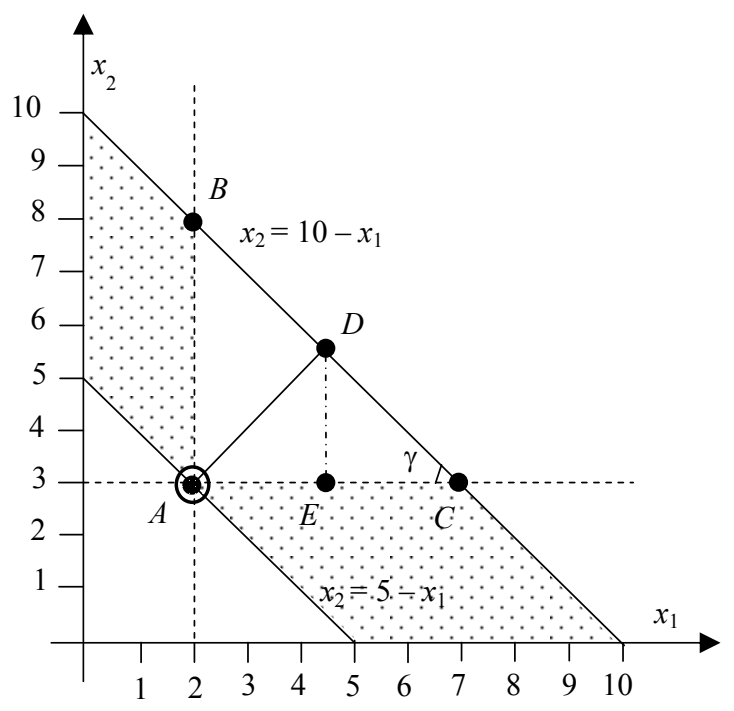

Рис. 1. Линии уровня 5 и 10

Кратчайшее расстояние из точки $A$ до прямой $x_{2}=10-x_{1}$ представляет собой длину перпендикуляра $A D$. Таким образом, при переходе из точки $A$ в точку $D$ изменение аргументов будет наименьшим. Изменение первого аргумента $\left(\Delta x_{1}\right)$ равно длине отрезка $A E$, изменение второго аргумента $\left(\Delta x_{2}\right)-$ отрезка $D E\left(A D^{2}=A E^{2}+D E^{2}\right)$.

Задача оптимизации при этом может быть представлена в виде задачи квадратичного программирования [11-12]:

$$
\begin{aligned}
& f\left(\Delta x_{1}, \Delta x_{2}\right)=\Delta x_{1}^{2}+\Delta x_{2}^{2} \rightarrow \min , \\
& 2+\Delta x_{1}+3+\Delta x_{2}=10 .
\end{aligned}
$$

Для решения данной задачи могут быть использованы методы нелинейной оптимизации: множителей Лагранжа, метод штрафов $[13,14]$. В результате будут получены следующие значения приращений: $\Delta x_{1}=2,5, \Delta x_{2}=2,5$. Таким образом, новые величины аргументов:

$$
\begin{aligned}
& x_{1}^{1}=2+2,5=4,5 ; \\
& x_{2}^{1}=3+2,5=5,5 .
\end{aligned}
$$

Из рис. 1 можно увидеть, что угол ADE равен углу ECD. Поскольку тангенс угла равен угловому коэффициенту, то

$$
\frac{A E}{D E}=-\rho,
$$

где $\rho-$ коэффициент угла наклона прямой.

Для рассматриваемого примера $\rho=-1$. Следовательно, для определения значений приращений с наименьшим изменением необходимо решить систему:

$$
\left\{\begin{array}{l}
\frac{\Delta x_{1}}{\Delta x_{2}}=1 \\
2+\Delta x_{1}+3+\Delta x_{2}=10 .
\end{array}\right.
$$

Подставляя во второе уравнение $\Delta x_{1}=\Delta x_{2}$, по-

$$
\begin{aligned}
& 2+\Delta x_{2}+3+\Delta x_{2}=10 \\
& \Delta x_{2}=2,5 ; \\
& \Delta x_{1}=2,5 .
\end{aligned}
$$

Рассмотрим случай при большем числе аргументов. Пусть функция имеет вид

$$
y=c_{1} x_{1}+c_{2} x_{2}+\ldots c_{n} x_{n}
$$

где $c$ - константы.

Тогда определение аргументов с наименьшим приращением будет выполнено путем решения системы:

$$
\left\{\begin{array}{l}
\frac{\Delta x_{i}}{\Delta x_{k}}=\frac{c_{i}}{c_{k}}, i=1 \ldots n, i \neq k ; \\
c_{1}\left(x_{1}^{0}+\Delta x_{1}\right)+c_{2}\left(x_{2}^{0}+\Delta x_{2}\right)+\ldots c_{n}\left(x_{n}^{0}+\Delta x_{n}\right)=y^{1},
\end{array}\right.
$$

где $k$ - номер аргумента, который принимается за базовый.

\section{Кратная модель}

При кратной зависимости модель имеет следующий вид (например, рентабельность определяется как отношение прибыли к затратам):

$$
y=\frac{x_{2}}{x_{1}} .
$$

Пусть начальные значения равны: $y^{0}=2, x_{1}^{0}=5$, $x_{2}^{0}=10$. Необходимо определить значения $x_{1}^{1}, x_{2}^{1}$, при которых значение функции равно 4.

Задача квадратичного программирования имеет вид

$$
\begin{aligned}
& f\left(\Delta x_{1}, \Delta x_{2}\right)=\Delta x_{1}^{2}+\Delta x_{2}^{2} \rightarrow \min \\
& \frac{\left(10+\Delta x_{2}\right)}{\left(5+\Delta x_{1}\right)}=4 .
\end{aligned}
$$

Решение задачи: $\Delta x_{1}=-2,353, \Delta x_{2}=0,588$, $x_{1}^{1}=2,647, x_{2}^{1}=10,588$.

На рис. 2 представлены линии уровня 2 и 4.

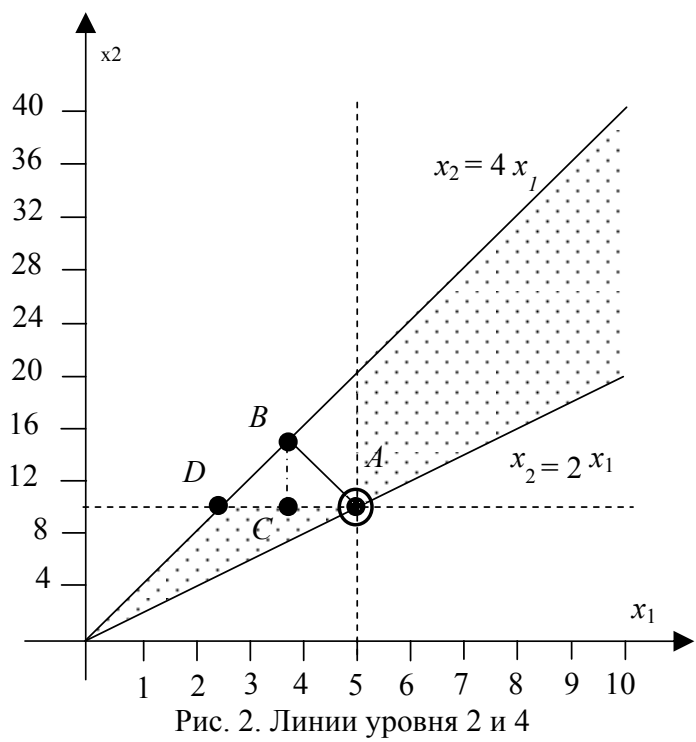

Начальные значения аргументов образуют точку $A$. Наименьшее расстояние из этой точки до прялучим 
мой $x_{2}=4 x_{1}-$ это длина перпендикуляра $A B$. Высота $B C$ образует два подобных треугольника. Следовательно, справедливо соотношение

$$
\frac{A C}{B C}=-4 \text {. }
$$

Тогда система будет иметь вид

$$
\left\{\begin{array}{l}
\frac{\Delta x_{1}}{\Delta x_{2}}=-4 \\
\frac{10+\Delta x_{2}}{5+\Delta x_{1}}=4
\end{array}\right.
$$

Решение системы: $\Delta x_{1}=-2,353, \Delta x_{2}=0,588$.

Мультипликативная модель

Наконец, рассмотрим мультипликативную зависимость (например, выручка от продажи товара равна произведению цены и количества):

$$
y=x_{1} \cdot x_{2} .
$$

Примем начальные условия задачи: $y^{0}=10$, $x_{1}^{0}=5, x_{2}^{0}=2$. Необходимо определить значения $x_{1}^{1}$, $x_{2}^{1}$, при которых $y^{1}$ равно 20 .

Задача квадратичного программирования:

$$
\begin{aligned}
& f\left(\Delta x_{1}, \Delta x_{2}\right)=\Delta x_{1}^{2}+\Delta x_{2}^{2} \rightarrow \min \\
& \left(5+\Delta x_{1}\right)\left(2+\Delta x_{2}\right)=20 .
\end{aligned}
$$

Решение задачи: $\Delta x_{1}=0,837, \Delta x_{2}=1,426$, $x_{1}^{1}=5,837, x_{2}^{1}=3,426$.

Рассмотрим линии уровня 10 и 20 (рис. 3). Точка А соответствует начальным значениям аргументов. Определение точки графика $x_{2}=20 / x_{1}$ таким образом, чтобы изменения аргументов были минимальны, может быть выполнено с использованием уравнения касательной:

$$
k=f^{\prime}\left(x_{0}\right)\left(x-x_{0}\right)+f\left(x_{0}\right),
$$

где $x_{0}$ - точка функции $f(x)$, к которой строится касательная.

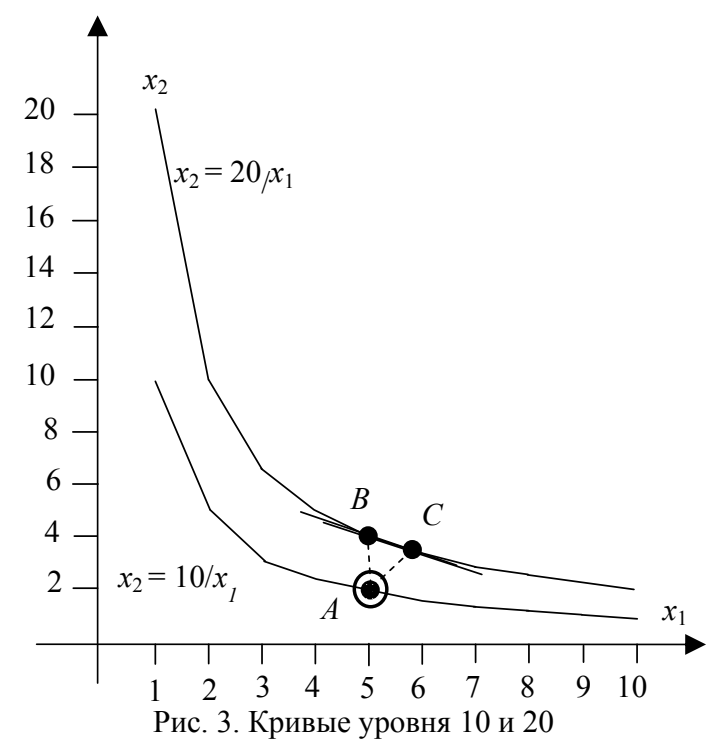

Уравнение касательной к точке $B$ будет иметь вид

$$
x_{2}=\frac{-20}{5^{2}}\left(x_{1}-5\right)+\frac{20}{5}=-0,8 x_{1}+8 .
$$

Поиск наименьших изменений аргументов для перехода в точку на прямой может быть выполнен с помощью решения системы уравнения:

$$
\left\{\begin{array}{l}
\frac{\Delta x_{1}}{\Delta x_{2}}=\frac{y^{1}}{\left(x_{1}^{0}\right)^{2}}=0,8 ; \\
0,8\left(5+\Delta x_{1}\right)+\left(2+\Delta x_{2}\right)=8 .
\end{array}\right.
$$

Отношение приращений аргументов будет равно угловому коэффициенту в уравнении касательной (со знаком «минус»). Решение системы: $\Delta x_{1}=0,976$, $\Delta x_{2}=1,22, x_{1}^{1}=5,976, x_{2}^{1}=3,22$.

Далее необходимо построить уравнение касательной к новой точке $\left(x_{1}^{1}=5,976, x_{2}^{1}=3,22\right)$ и выполнить поиск новой точки с минимальным изменением аргументов.

Таким образом, алгоритм нахождения решения в случае мультипликативной модели включает следующие шаги:

1. Установка начальных значений:

$$
i=0, x_{1}(i)=x_{1}^{0}, x_{2}(i)=x_{2}^{0} .
$$

2. Построение уравнения касательной к точке $x_{1}(i)$ функции $x_{2}=\frac{y^{1}}{x_{1}}$.

3. Поиск точки $x_{1}(i+1), x_{2}(i+1)$ на касательной, до которой расстояние от исходной точки $x_{1}^{0}$, $x_{2}^{0}$ будет минимальным.

4. Проверка выполнения условия останова: если изменение положения точки меньше заданной точности $\varepsilon\left(\delta=\sqrt{\left(x_{1}(i+1)-x_{1}(i)\right)^{2}+\left(x_{2}(i+1)-x_{2}(i)\right)^{2}}<\varepsilon\right)$, то алгоритм завершается, иначе осуществляется переход на шаг $2(i=i+1)$.

В таблице представлены результаты выполнения итераций для рассматриваемого примера $(\varepsilon=0,003)$.

Результаты выполнения итераций
\begin{tabular}{|c|c|c|c|c|}
\hline$i$ & $x_{1}(i)$ & $x_{2}(i)$ & $-f^{\prime}\left(x_{1}(i)\right)$ & $\delta$ \\
\hline 0 & 5 & 2 & 0,8 & - \\
\hline 1 & 5,976 & 3,22 & 0,56 & 1 \\
\hline 2 & 5,807 & 3,441 & 0,593 & 0,278 \\
\hline 3 & 5,844 & 3,423 & 0,586 & 0,041 \\
\hline 4 & 5,835 & 3,425 & 0,587 & 0,009 \\
\hline 5 & 5,838 & 3,428 & 0,587 & 0,004 \\
\hline 6 & 5,837 & 3,426 & - & 0,002 \\
\hline
\end{tabular}

Пример формирования рейтинговой оценки

В качестве примера рассмотрим также задачу формирования рейтинга группы онлайновой соци- 
альной сети. Интегральная оценка вычисляется по формуле:

$$
R_{j}=0,701 x_{1 j}+0,24 x_{2 j}+0,059 x_{3 j},
$$

где $x_{1 j}$ - нормированное значение числа подписчиков $j$-й группы; $x_{2 j}$ - нормированное значение показателя популярности $j$-й группы; $x_{3 j}$ - нормированное значение показателя активности $j$-й группы.

Для выбранной группы значения нормированных величин равны: $x_{1}^{0}=0,313, x_{2}^{0}=0,004, x_{3}^{0}=0,029$. Тогда интегральная оценка составит:

$$
R=0,701 \cdot 0,313+0,24 \cdot 0,004+0,059 \cdot 0,029=0,222 .
$$

Пусть необходимо определить такие новые значения $x$, которые обеспечат значение рейтинговой оценки, равное 0,3, при минимальном изменении аргументов.

Система уравнений имеет вид:

$$
\left\{\begin{array}{l}
\frac{\Delta x_{1}}{\Delta x_{2}}=\frac{c_{1}}{c_{2}}=\frac{0,701}{0,24} ; \\
\frac{\Delta x_{3}}{\Delta x_{2}}=\frac{c_{3}}{c_{2}}=\frac{0,059}{0,24} ; \\
0,701\left(0,313+\Delta x_{1}\right)+0,24\left(0,004+\Delta x_{2}\right)+ \\
+0,059\left(0,029+\Delta x_{3}\right)=0,3 .
\end{array}\right.
$$

Решение системы: $\Delta x_{1}=0,099, \Delta x_{2}=0,034$, $\Delta x_{3}=0,008$. Таким образом,

$$
\begin{aligned}
& x_{1}^{1}=0,313+0,099=0,412 ; \\
& x_{2}^{1}=0,004+0,034=0,038 ; \\
& x_{3}^{1}=0,029+0,008=0,037 .
\end{aligned}
$$

\section{Заключение}

В статье рассмотрено решение обратных задач экономического анализа с помощью обратных вычислений путем минимизации приращений аргументов. Модификация классической схемы решения заключается в использовании в качестве отношения коэффициентов относительной важности углового коэффициента линии установленного уровня. Представлена графическая интерпретация используемых соотношений. По сравнению с решением оптимизационных задач нелинейного программирования представленный подход является более простым в компьютерной реализации: решение задачи сводится к решению системы алгебраических уравнений. Рассмотрен случай аддитивной, кратной и мультипликативной зависимости между аргументами. Также в статье представлен пример формирования рейтинговой оценки с заданным значением интегрального показателя. Результаты вычислений совпали с результатами, полученными путем решения задачи нелинейного программирования с использованием оптимизационных методов.
С помощью представленных методов может быть получено решение обратных задач при отсутствии экспертных оценок о важности аргументов функции и направлении изменения показателей и минимальном изменении исходных данных.

Также рассмотренный подход может быть использован для решения отдельных задач квадратичного программирования с одним ограничением [15].

\section{Литература}

1. Одинцов Б.Е. Обратные вычисления в формировании экономических решений. - М.: Финансы и статистика, 2004. $-256 \mathrm{c}$.

2. Дик В.В. Методология формирования решений в экономических системах и инструментальные среды их поддержки. - М.: Финансы и статистика, 2001. - 300 с.

3. Одинцов Б.Е. Итерационный метод оптимизации управления предприятиями средствами обратных вычислений / Б.Е. Одинцов, А.Н. Романов // Вестник Финансового ун-та. - 2014. - № 2. - С. 60-73.

4. Виштак О.В. Использование технологии обратных вычислений при мониторинге качества дополнительного образования в вузе / О.В. Виштак, И.А. Штырова // Вестник Астрахан. гос. техн. ун-та. - 2014. - № 2. - С. 67-73.

5. Бармина Е.А. Мониторинг качества коммерческой организации. Структурирование показателей. Применение когнитивных карт / Е.А. Бармина, И.Ю. Квятковская // Вестник Астрахан. гос. техн. ун-та. - 2010. - № 2. - С. 15-20.

6. Мартьянова А.В. Управление эффективностью банка на базе обратных вычислений // Вестник магистратуры. - 2015. - №6(45). - С. 77-79.

7. Одинцов Б.Е. Когнитивные древовидные структуры в управлении слабоформализованными социальноэкономическими процессами // Информатизация образования и науки. -2017 . - № 2. - С. 46-56.

8. Блюмин С.Л. // Обратные задачи в лагранжевом анализе конечных изменений / С.Л. Блюмин, Г.С. Боровкова, А.С. Сысоев // Современные проблемы горно-металлургического комплекса. Наука и производство: матер. Тринадцатой Всерос. науч.-практ. конф. - Старый Оскол, 2016. - T. 2. - C. 6-10.

9. Грибанова Е.Б. Стохастические алгоритмы решения обратных задач с ограничениями // Доклады ТУСУР. 2016. - № 4. - С. 112-116.

10. Одинцов Б.Е. Управление с учетом «золотых» пропорций плановых показателей // Управленческие науки в современном мире. - 2016. - № 1. - С. 43-47.

11. Грибанова Е.Б. Методы решения обратных задач экономического анализа // Корпоративные финансы. 2016. - № 1. - С. 119-130.

12. Сіницький М. Є. До питання розв'язку обернених задач економічного спрямування // Науковий вісник Національної академії статистики, обліку та аудиту. 2018. - № 1. - C. 195-202.

13. Мицель А.А. Методы оптимизации: учеб. пособие / А.А. Мицель, А.А. Шелестов. - Томск: Изд-во ТУСУРa, 2004. - $256 \mathrm{c}$.

14. Карманов В.Г. Математическое программирование: учеб. пособие. - М.: Наука, 1989. - 263 с.

15. Грибанова Е.Б. Решение задачи оптимизации закупок с помощью обратных вычислений // Экономический анализ: теория и практика. - 2018. - № 3. - С. 586-596. 


\section{Грибанова Екатерина Борисовна}

Канд. техн. наук, доцент каф. автоматизированных систем управления (АСУ) Томского государственного ун-та систем управления и радиоэлектроники (ТУСУР)

Ленина пр-т, д. 40, г. Томск, Россия, 634050

Тел.: +7 (382-2) 70-15-36

Эл. почта: katag@yandex.ru

Gribanova E.B.

Methods for solving inverse problems of economic analysis by minimizing argument increments

The article describes the method of solving inverse problems of economic analysis by minimizing the increments of arguments. A simpler solution was obtained in comparison with the use of optimization methods of nonlinear programming. Additive, multiplicative and multiple models are considered. The task of rating formation is given as an example.

Keywords: inverse computations, optimization, quadratic programming, rating.

doi: $10.21293 / 1818-0442-2018-21-2-95-99$

\section{References}

1. Odincov B.E. Obratnye vychislenija $v$ formirovanii jeko-nomicheskih reshenij [Inverse computations in forming of economic decisions]. Moscow, Finansy i statistika Publ., 2004. 256 p.

2. Dik V.V. Metodologija formirovanija reshenij v jekonomicheskih sistemah i instrumental'nye sredy ih podderzhki [Methodology of decision-making in economic systems and instrumental environment of their support]. Moscow, Finansy i statistika Publ., 2001, 300 p.

3. Odintsov B.E., Romanov A.N. An iterative method of optimization of enterprise management by means of inverse calcalations. The bulletin of the financial university, 2014, no. 2, pp. 60-73. (In Russ.)

4. Vishtak O.V., Shtyrova I.A. The use of technology of inverse calculations when monitoring the quality of additional education at the University. The bulletin of Astrakhan state technical university, 2014, no. 2, pp. 67-73.

5. Barmina E.A., Kvjatkovskaja I.Ju. Quality monitoring of a commercial organization. The structuring of indicators. Application of cognitive maps. The bulletin of Astrakhan state technical university, 2010, no. 2, pp. 15-20.
6. Mart'janova A.V. The performance management of the bank using the inverse calculation. Bulletin of the magistracy, 2015 , no. 6 , pp. $77-79$.

7. Odincov B.E. Cognitive tree structures in the management of weakly formalized socio-economic processes. Informatization of education and science, 2017, no. 2, pp. 46-56.

8. Bljumin S.L., Borovkova G.S., Sysoev A.S. Obratnye zadachi v lagranzhevom analize konechnyh izmene-nij [The inverse problem in Lagrangian analysis of the final changes]. Sovremennye problemy gorno-metallurgicheskogo kompleksa. Nauka i proizvodstvo. Materialy trinadcatoj Vserossijskoj nauchno-prakticheskoj konferencii [Modern problems of mining and metallurgical complex. Science and production. Proc. of the thirteenth All-Russian scientific-practical conference]. Staryj Oskol, 2016, vol. II, pp. 6-10.

9. Gribanova E.B. Stochastic algorithms for solving the economic analysis inverse problems with constraints. Proceedings of TUSUR University, 2016, no. 4, pp. 112-116.

10. Odintsov B.E. Management taking into account the «golden» proportions of the planned indicators. Managerial sciences in the modern world, 2016, no. 1, pp. 43-47.

11. Gribanova E.B. Methods for solving inverse problems of economic analysis. Corporate Finance, 2016, no. 1, pp. 119-130.

12. Sinic'kij M. $€$. To the solution of inverse problems of economic direction. Scientific Bulletin of National Academy of statistics, accounting and audit, 2018, no. 1-2, pp. 195-202.

13. Micel' A.A., Shelestov A.A. Metody optimizacii: uchebnoe posobie [Optimization method]. Tomsk, TUSUR Publ., 2004, 256 p.

14. Karmanov V.G. Matematicheskoe programmirovanie: uchebnoe posobie [Mathematical programming]. Moscow, Nauka Publ., 1989, 263 p.

15. Gribanova E.B. Solving the procurement optimization problem by means of inverse computation. Economic analysis: theory and practice, 2018, no. 3, pp. 586-596.

\section{Ekaterina B. Gribanova}

Candidate of Engineering Sciences, Assistant Professor, Department of Automated Control System, Tomsk State University of Control Systems and Radioelectronics (TUSUR) 40, Lenina pr., Tomsk, Russia, 634050

Phone: 7 (382-2) 70-15-36

Email:katag@yandex.ru 